\title{
Sums of one prime and two prime squares
}

by

\author{
Hongze Li (Shanghai)
}

\section{Introduction. Let}

$$
\begin{aligned}
& \mathcal{A}=\{n: n \in \mathbb{N}, n \equiv 1(\bmod 2), n \not \equiv 2(\bmod 3)\}, \\
& \mathcal{C}=\{n: n \in \mathbb{N}, n \equiv 3(\bmod 24), n \not \equiv 0(\bmod 5)\} .
\end{aligned}
$$

In 1938 Hua [3] proved that almost all $n \in \mathcal{A}$ are representable as sums of two squares of primes and a $k$ th power of a prime for odd $k$, and almost all $n \in \mathcal{C}$ are representable as sums of two squares of primes and a $k$ th power of a prime for even $k$. The natural question then becomes: how good a bound can we get on the possible exceptional sets? Let $E_{k}(N)$ denote the number of exceptions up to $N$ for the problem with $k$ th power of a prime. Hua's result actually shows that $E_{k}(N) \ll N(\log N)^{-A}$ for some positive constant $A$. Later Schwarz [6] refined Hua's result to show that

$$
E_{k}(N) \ll N(\log N)^{-A} \quad \text { for any } A>0 .
$$

In 1993 Leung and Liu [4] improved this to $E_{k}(N) \ll N^{1-\delta}$ for some fixed $\delta>0$.

For the special case $k=1$,

$$
n=p_{1}+p_{2}^{2}+p_{3}^{2} .
$$

In 2004 Wang [7] proved that $E_{1}(N) \ll N^{13 / 30+\varepsilon}$. In 2006 Wang and Meng [8] improved it to $E_{1}(N) \ll N^{5 / 12+\varepsilon}$. In this note we shall prove the following result.

TheOREm. Let $\varepsilon>0$ be given. Then for all large $N$ we have

$$
E_{1}(N) \ll N^{5 / 14+\varepsilon} .
$$

The improvement is due to the application of a sieve method. The basic idea is to show that the argument of [2] used for four squares of primes can

2000 Mathematics Subject Classification: 11P32, 11P05, 11P55.

Key words and phrases: exceptional set, sieve method, circle method.

This work was supported by the National Natural Science Foundation of China (10471090 and 10771135). 
be adapted to work for a prime and two squares of primes to give the same size exceptional set. We can therefore quote much from the proof in [2], sketching the necessary changes.

2. Outline and preliminary results. To prove the Theorem, it suffices to estimate the number of exceptional integers in the set $\mathcal{B}:=$ $\mathcal{A} \cap(N / 2, N]$. Here $N$ is our main parameter, which we assume to be "sufficiently large". We write

$$
P=N^{1 / 7-\varepsilon}, \quad Q=N P^{-1} \mathcal{L}^{-100}, \quad M=N \mathcal{L}^{-9}, \quad \mathcal{L}=\log N .
$$

We use $c$ and $\varepsilon$ to denote an absolute constant and a sufficiently small positive number, not necessarily the same at each occurrence.

Let

$$
\mathfrak{M}=\bigcup_{1 \leq q \leq P} \bigcup_{\substack{1 \leq a \leq q \\(a, q)=1}}\left[\frac{a}{q}-\frac{1}{q Q}, \frac{a}{q}+\frac{1}{q Q}\right] .
$$

These are the major arcs, and so the minor arcs $\mathfrak{m}$ are given by

$$
\mathfrak{m}=\left[\frac{1}{Q}, 1+\frac{1}{Q}\right] \backslash \mathfrak{M} .
$$

Let us begin with

$$
\sum_{\substack{p_{1}+p_{2}^{2}+m^{2}=n \\ M<p_{1}, p_{2}^{2}, m^{2} \leq N}}\left(\log p_{1}\right)\left(\log p_{2}\right) \rho(m)=\int_{0}^{1} f(\alpha) g(\alpha) h(\alpha) e(-\alpha n) d \alpha,
$$

in which $e(x)=\exp (2 \pi i x)$ and

$$
\begin{aligned}
& f(\alpha)=\sum_{M<p \leq N}(\log p) e(\alpha p), \quad g(\alpha)=\sum_{M<p^{2} \leq N}(\log p) e\left(\alpha p^{2}\right), \\
& h(\alpha)=\sum_{M<m^{2} \leq N} \rho(m) e\left(\alpha m^{2}\right) .
\end{aligned}
$$

Here $\rho(m)$ satisfy

$$
\rho(m) \leq\left\{\begin{array}{ll}
1 & \text { if } m \text { is prime, } \\
0 & \text { otherwise, }
\end{array} \quad \text { and } \quad \sum_{m \leq X} \rho(m) \gg X \mathcal{L}^{-1}\right.
$$

for $N^{1 / 4} \leq X \leq N^{1 / 2}$. This means that $\rho$ is a non-trivial lower bound for the characteristic function of the set of primes in $\left[M^{1 / 2}, N^{1 / 2}\right]$.

The new idea introduced in Section 3 of [2], and which we use here, is as follows. The maximum saving we can make for $g(\alpha)$ on the minor arcs with our current knowledge is $N^{1 / 16}$, but this can be increased to $N^{1 / 14}$ for $h(\alpha)$. The final exponent for the exceptional set is then $\frac{1}{2}-2 \cdot \frac{1}{14}=\frac{5}{14}$ using an argument of Wooley that motivates (4.3)-(4.6) below. 
Let $\theta(m, \alpha)$ be the function which is 1 except when there exist integers $a$ and $q$ such that

$|q \alpha-a|<Q^{-1},(a, q)=1, q \leq P, \quad(m, q)$ is divisible by a prime $p \geq N^{1 / 14}$, in which case $\theta(m, \alpha)=0$. Define

$$
k(\alpha)=\sum_{M<m^{2} \leq N} \rho(m) \theta(m, \alpha) e\left(\alpha m^{2}\right), \quad l(\alpha)=h(\alpha)-k(\alpha) .
$$

It is easy to see that, for $\alpha \in \mathfrak{m}, h(\alpha)=k(\alpha)$ and

$$
l(\alpha) \ll N^{3 / 7}
$$

for all $\alpha$.

For a positive integer $k$ and $\chi \bmod q$, define

$$
C_{k}(\chi, a)=\sum_{h=1}^{q} \bar{\chi}(h) e\left(a h^{k} / q\right), \quad C_{k}(q, a)=C_{k}\left(\chi_{0}, a\right) .
$$

Here $\chi_{0}$ is the principal character modulo $q$.

If $\chi_{1}, \chi_{2}, \chi_{3}$ are characters modulo $q$, then let

and

$$
\begin{array}{r}
B\left(n, q ; \chi_{1}, \chi_{2}, \chi_{3}\right) \\
=\frac{1}{\phi^{3}(q)} \sum_{\substack{a=1 \\
(a, q)=1}}^{q} C_{1}\left(\chi_{1}, a\right) C_{2}\left(\chi_{2}, a\right) C_{2}\left(\chi_{3}, a\right) e(-a n / q),
\end{array}
$$

$$
A(q)=B\left(n, q ; \chi_{0}, \chi_{0}, \chi_{0}\right), \quad \mathfrak{S}(n, X)=\sum_{q \leq X} A(q) .
$$

Lemma 1 (Lemma 7.1 of [8]). For $n \in \mathcal{A}$, we have

$$
\mathfrak{S}(n, X) \gg 1 \text {. }
$$

Lemma $2($ Lemma 3.1 of $[8])$. Let $\chi_{j}\left(\bmod r_{j}\right)$ with $j=1,2,3$ be primitive characters, $r_{0}=\left[r_{1}, r_{2}, r_{3}\right]$, and $\chi_{0}$ the principal character modulo $q$. Then

$$
\sum_{\substack{q \leq x \\ r_{0} \mid q}}\left|B\left(n, q ; \chi_{1} \chi_{0}, \chi_{2} \chi_{0}, \chi_{3} \chi_{0}\right)\right| \ll r_{0}^{-1 / 2+\varepsilon}(\log x)^{10} .
$$

Lemma 3 (Theorem 1.1 of [1]). Let $\ell \in \mathbb{N}, R, T, X \geq 1$ and $\kappa:=1 / \log X$. Then there is an absolute positive constant $c$ such that

$$
\sum_{\substack{r \sim R \\ \ell \mid r}} \sum_{\chi(\bmod r)}^{*} \int_{-T}^{T}\left|\sum_{X \leq n \leq 2 X} \frac{\Lambda(n) \chi(n)}{n^{\kappa+i \tau}}\right| d \tau \ll\left(\ell^{-1} R^{2} T X^{11 / 20}+X\right)(\log R T X)^{c},
$$

where $\sum_{\chi(\bmod r)}^{*}$ means summation over the primitive characters modulo $r$. The implied constant is absolute. 
3. The major arcs. Let

$$
\begin{aligned}
f^{*}(\alpha) & =\frac{C_{1}(q, a)}{\phi(q)} \sum_{M<m \leq N} e(\beta m), \\
g^{*}(\alpha) & =\frac{C_{2}(q, a)}{\phi(q)} \sum_{M<m^{2} \leq N} e\left(\beta m^{2}\right), \\
k^{*}(\alpha) & =\frac{C_{2}(q, a)}{\phi(q)} \sum_{M<m^{2} \leq N} \varrho(m) e\left(\beta m^{2}\right),
\end{aligned}
$$

where $\varrho(m)$ is defined in (4.3) of [2]. We now consider

$$
\int_{\mathfrak{M}} f(\alpha) g(\alpha) k(\alpha) e(-\alpha n) d \alpha-\int_{\mathfrak{M}} f^{*}(\alpha) g^{*}(\alpha) k^{*}(\alpha) e(-\alpha n) d \alpha,
$$

which we think of as the error term over $\mathfrak{M}$.

Define

$$
\begin{aligned}
W_{1}(\chi, \beta) & =\sum_{M<p \leq N}(\log p) \chi(p) e(\beta p)-D(\chi) \sum_{M<m \leq N} e(\beta m), \\
W_{2}(\chi, \beta) & =\sum_{M<p^{2} \leq N}(\log p) \chi(p) e\left(\beta p^{2}\right)-D(\chi) \sum_{M<m^{2} \leq N} e\left(\beta m^{2}\right), \\
W^{\sharp}(\chi, \beta) & =\sum_{M<m^{2} \leq N} \rho(m) \chi(m) e\left(\beta m^{2}\right)-D(\chi) \sum_{M<m^{2} \leq N} \varrho(m) e\left(\beta m^{2}\right),
\end{aligned}
$$

where $D(\chi)$ is 1 or 0 according as $\chi$ is principal or not.

Similar to (4.1) of [2], we can write the $f(\alpha), g(\alpha)$ and $k(\alpha)$ as

$$
\begin{aligned}
& f\left(\frac{a}{q}+\beta\right)=\frac{C_{1}(q, a)}{\phi(q)} \sum_{M<m \leq N} e(\beta m)+\frac{1}{\phi(q)} \sum_{\chi \bmod q} C_{1}(\chi, a) W_{1}(\chi, \beta), \\
& g\left(\frac{a}{q}+\beta\right) \\
& \quad=\frac{C_{2}(q, a)}{\phi(q)} \sum_{M<m^{2} \leq N} e\left(\beta m^{2}\right)+\frac{1}{\phi(q)} \sum_{\chi \bmod q} C_{2}(\chi, a) W_{2}(\chi, \beta), \\
& k\left(\frac{a}{q}+\beta\right) \\
& =\frac{C_{2}(q, a)}{\phi(q)} \sum_{M<m^{2} \leq N} \varrho(m) e\left(\beta m^{2}\right)+\frac{1}{\phi(q)} \sum_{\chi \bmod q} C_{2}(\chi, a) W^{\sharp}(\chi, \beta) .
\end{aligned}
$$

So we can use (3.5)-(3.7) to express the difference in (3.4) as a linear combination of error terms involving $f^{*}(\alpha), g^{*}(\alpha)$ and $k^{*}(\alpha)$, and $W_{1}(\chi, \beta)$, 
$W_{2}(\chi, \beta)$ and $W^{\sharp}(\chi, \beta)$. In these error terms, the most troublesome is

$$
\sum_{q \leq P} \sum_{\chi_{1} \bmod } \sum_{\chi_{2} \bmod } \sum_{\chi_{3} \bmod q} B\left(n, q ; \chi_{1}, \chi_{2}, \chi_{3}\right) J\left(n, q, \chi_{1}, \chi_{2}, \chi_{3}\right) .
$$

Here $B\left(n, q ; \chi_{1}, \chi_{2}, \chi_{3}\right)$ is defined in $(2.10)$, and

$$
J\left(n, q, \chi_{1}, \chi_{2}, \chi_{3}\right)=\int_{-1 / q Q}^{1 / q Q} W^{\sharp}\left(\chi_{3}, \beta\right) W_{1}\left(\chi_{1}, \beta\right) W_{2}\left(\chi_{2}, \beta\right) e(-\beta n) d \beta .
$$

Suppose $\chi_{j}^{*} \bmod r_{j}, r_{j} \mid q$, is the primitive character inducing $\chi_{j}$. If $\chi \bmod q$, $q \leq P$, is induced by a primitive character $\chi^{*} \bmod r, r \mid q$, we have

$$
\begin{aligned}
& W_{j}(\chi, \beta)=W_{j}\left(\chi^{*}, \beta\right), \quad j=1,2, \\
& W^{\sharp}(\chi, \beta)=W^{\sharp}\left(\chi^{*}, \beta\right)+O\left(r^{-2} N^{13 / 28}\right),
\end{aligned}
$$

where the error term comes from the integers in the set

$$
\left\{m^{2} \in[M, N]:(m, q)>1,(m, r)=1, \rho(m) \neq 0\right\} .
$$

When $r \leq P N^{-3 / 28}<N^{1 / 28}$, this set contains $\ll N^{1 / 2-3 / 28} \ll r^{-2} N^{13 / 28}$ integers; when $r>P N^{-3 / 28}$, it is empty.

By Cauchy's inequality,

$$
J\left(n, q, \chi_{1}, \chi_{2}, \chi_{3}\right) \ll\left(W^{\sharp}\left(\chi_{3}^{*}\right)+r_{3}^{-2} N^{13 / 28}\right) W_{1}\left(\chi_{1}^{*}\right) W_{2}\left(\chi_{2}^{*}\right),
$$

where for a character $\chi \bmod r$,

$$
\begin{aligned}
W^{\sharp}(\chi) & =\max _{|\beta| \leq 1 / r Q}\left|W^{\sharp}(\chi, \beta)\right|, \\
W_{j}(\chi) & =\left(\int_{-1 / r Q}^{1 / r Q}\left|W_{j}(\chi, \beta)\right|^{2} d \beta\right)^{1 / 2}, \quad j=1,2 .
\end{aligned}
$$

By (3.11), the quantity (3.8) is

$$
\begin{aligned}
\ll \sum_{r_{1} \leq P} \sum_{\chi_{1}}^{*} \sum_{r_{2} \leq P} \sum_{\chi_{2}}^{*} \sum_{r_{3} \leq P} \sum_{\chi_{3}}^{*}\left(W^{\sharp}\left(\chi_{3}\right)+r_{3}^{-2} N^{13 / 28}\right) \\
\times W_{1}\left(\chi_{1}\right) W_{2}\left(\chi_{2}\right) B\left(n, \chi_{1}, \chi_{2}, \chi_{3}\right) .
\end{aligned}
$$

Here $\sum_{r_{j}} \sum_{\chi_{j}}^{*}$ denotes summation over the primitive characters to moduli $r_{j} \leq P$, and

$$
B\left(n, \chi_{1}, \chi_{2}, \chi_{3}\right)=\sum_{\substack{q \leq P \\ r_{0} \mid q}}\left|B\left(n, q ; \chi_{1} \chi_{0}, \chi_{2} \chi_{0}, \chi_{3} \chi_{0}\right)\right|,
$$

where $r_{0}=\left[r_{1}, r_{2}, r_{3}\right]$ and $\chi_{0}$ is the principal character modulo $q$.

By Lemma 2 we have

$$
B\left(n, \chi_{1}, \chi_{2}, \chi_{3}\right) \ll r_{0}^{-1 / 2+\varepsilon} \mathcal{L}^{10},
$$


and by Lemma 2.4 of [5],

$$
\sum_{r \leq R} \sum_{\chi}^{*}[r, d]^{-1 / 2+\varepsilon} W_{2}(\chi) \ll d^{-1 / 2+\varepsilon} \mathcal{L}^{c}
$$

whenever $R \leq N^{1 / 6-\varepsilon}$. Thus the sum in (3.13) does not exceed

$$
\mathcal{L}^{c} \sum_{r_{1} \leq P} \sum_{\chi_{1}}^{*} \sum_{r_{3} \leq P} \sum_{\chi_{3}}^{*}\left[r_{1}, r_{3}\right]^{-1 / 2+\varepsilon}\left(W^{\sharp}\left(\chi_{3}\right)+r_{3}^{-2} N^{13 / 28}\right) W_{1}\left(\chi_{1}\right) .
$$

Following Section 6 of [5], but using Lemma 3 instead of Theorem 4.1 of [5], with a few changes, we get

$$
\sum_{r_{1} \leq P} \sum_{\chi_{1}}^{*}\left[r_{1}, r_{3}\right]^{-1 / 2+\varepsilon} W_{1}\left(\chi_{1}\right) \ll r_{3}^{-1 / 2+\varepsilon} N^{1 / 2} \mathcal{L}^{c},
$$

so (3.14) does not exceed

$$
\begin{aligned}
& \mathcal{L}^{c} N^{1 / 2} \sum_{r_{3} \leq P} \sum_{\chi_{3}}^{*}\left(r_{3}^{-1 / 2+\varepsilon} W^{\sharp}\left(\chi_{3}\right)+r_{3}^{-5 / 2+\varepsilon} N^{13 / 28}\right) \\
& \ll \mathcal{L}^{c} N^{1 / 2} \sum_{r_{3} \leq P} \sum_{\chi_{3}} r_{3}^{-1 / 2+\varepsilon} W^{\sharp}\left(\chi_{3}\right)+N^{27 / 28+\varepsilon} .
\end{aligned}
$$

By the argument of page 8 of [2], if $\rho(m)$ satisfies conditions (i), (ii), (iv) and (v) in [2], then for any fixed $A>0$ we have

$$
\sum_{r_{3} \leq P} \sum_{\chi_{3}}^{*} r_{3}^{-1 / 2+\varepsilon} W^{\sharp}\left(\chi_{3}\right) \ll N^{1 / 2} \mathcal{L}^{-A-c} .
$$

Therefore, by (3.9)-(3.17) we have

$$
\sum_{q \leq P} \sum_{\chi_{1} \bmod } \sum_{q \chi_{2} \bmod q} \sum_{\chi_{3} \bmod q} B\left(n, q ; \chi_{1}, \chi_{2}, \chi_{3}\right) J\left(n, q, \chi_{1}, \chi_{2}, \chi_{3}\right)
$$

$$
\ll N \mathcal{L}^{-A}
$$

for any fixed $A>0$.

Hence the sum in (3.8) is $O\left(N \mathcal{L}^{-A}\right)$ for any fixed $A>0$. Similarly, the other error terms in (3.4) can be estimated in the same way, so the difference in $(3.4)$ is $O\left(N \mathcal{L}^{-A}\right)$.

By the standard major arcs techniques we have

$$
\int_{\mathfrak{M}} f^{*}(\alpha) g^{*}(\alpha) k^{*}(\alpha) e(-\alpha n) d \alpha=P_{0} \mathfrak{S}(n, P)(1+o(1)),
$$

where

$$
N \mathcal{L}^{-1} \ll P_{0}=\sum_{\substack{m_{1}+m_{2}^{2}+m_{3}^{2}=n \\ M<m_{1}, m_{2}^{2}, m_{3}^{2} \leq N}} \varrho\left(m_{3}\right) \ll N \mathcal{L}^{-1},
$$

by (4.4) of [2], and $\mathfrak{S}(n, P)$ is defined by $(2.11)$. 
By Lemma 1, (3.4) and (3.19)-(3.20) we obtain the following result:

Lemma 4. Suppose that $\rho(m)$ satisfies conditions (i), (ii), (iv) and (v) in [2]. Then for sufficiently large $n \in \mathcal{A}$, we have

$$
\int_{\mathfrak{M}} f(\alpha) g(\alpha) k(\alpha) e(-\alpha n) d \alpha \gg N \mathcal{L}^{-1} \text {. }
$$

4. Proof of Theorem. Let $\mathcal{E}(N)$ be the set of integers $n \in \mathcal{B}$ such that

$$
n \neq p_{1}+p_{2}^{2}+p_{3}^{2} \text {. }
$$

It is sufficient to prove that

$$
\mathcal{E}(N) \ll N^{5 / 14+\varepsilon} .
$$

Let $|\mathcal{E}(N)|$ denote the cardinality of $\mathcal{E}(N)$ and $Z(\alpha)$ be its generating function:

$$
Z(\alpha)=\sum_{n \in \mathcal{E}(N)} e(-\alpha n)
$$

Then by (2.2)-(2.6) we have

$$
\int_{0}^{1} f(\alpha) g(\alpha) h(\alpha) Z(\alpha) d \alpha \leq 0 .
$$

By Lemma 4, it follows that

$$
\int_{\mathfrak{M}} f(\alpha) g(\alpha) k(\alpha) Z(\alpha) d \alpha \gg|\mathcal{E}(N)| N \mathcal{L}^{-1} \text {. }
$$

Thus

$$
\begin{aligned}
\left|\int_{\mathfrak{M}} f(\alpha) g(\alpha) k(\alpha) Z(\alpha) d \alpha-\int_{0}^{1} f(\alpha) g(\alpha) h(\alpha) Z(\alpha) d \alpha\right| \\
=\left|\int_{\mathfrak{M}} f(\alpha) g(\alpha)(k(\alpha)-h(\alpha)) Z(\alpha) d \alpha-\int_{\mathfrak{m}} f(\alpha) g(\alpha) h(\alpha) Z(\alpha) d \alpha\right| \\
\gg|\mathcal{E}(N)| N \mathcal{L}^{-1} .
\end{aligned}
$$

By Lemma 1 of [2] and (2.8) we have

$$
\begin{aligned}
&|\mathcal{E}(N)| \ll \mathcal{L} N^{-1}\left(\int_{\mathfrak{M}}|f(\alpha) g(\alpha)(k(\alpha)-h(\alpha)) Z(\alpha)| d \alpha\right. \\
&\left.\quad+\int_{\mathfrak{m}}|f(\alpha) g(\alpha) h(\alpha) Z(\alpha)| d \alpha\right) \\
& \ll \mathcal{L} N^{-1} N^{3 / 7+\varepsilon / 5} \int_{0}^{1}|f(\alpha) g(\alpha) Z(\alpha)| d \alpha .
\end{aligned}
$$


Now we apply the device introduced by Wooley [9] and used by Harman and Kumchev [2], namely by Cauchy's inequality and Parseval's identity we have

$$
\int_{0}^{1}|f(\alpha) g(\alpha) Z(\alpha)| d \alpha \ll\left(\int_{0}^{1}|f(\alpha)|^{2} d \alpha\right)^{1 / 2}\left(\int_{0}^{1}|g(\alpha) Z(\alpha)|^{2} d \alpha\right)^{1 / 2} .
$$

It is easy to see that

$$
\begin{aligned}
& \int_{0}^{1}|f(\alpha)|^{2} d \alpha=\sum_{M<p \leq N}(\log p)^{2} \ll N \mathcal{L}, \\
& \int_{0}^{1}|g(\alpha) Z(\alpha)|^{2} d \alpha=\sum_{\substack{p_{1}^{2}+n_{1}=p_{2}^{2}+n_{2} \\
M<p_{i}^{2} \leq N, n_{i} \in \mathcal{E}(N)}}\left(\log p_{1}\right)\left(\log p_{2}\right) \\
& \ll N^{1 / 2+\varepsilon / 4}|\mathcal{E}(N)|+|\mathcal{E}(N)|^{2} N^{\varepsilon / 4} .
\end{aligned}
$$

Therefore

$$
\int_{0}^{1}|f(\alpha) g(\alpha) Z(\alpha)| d \alpha \ll N^{3 / 4+\varepsilon / 4}|\mathcal{E}(N)|^{1 / 2}+N^{1 / 2+\varepsilon / 2}|\mathcal{E}(N)| .
$$

So by (4.3)-(4.6) we have

$$
|\mathcal{E}(N)| \ll N^{5 / 28+\varepsilon / 2}|\mathcal{E}(N)|^{1 / 2} .
$$

From this we get

$$
|\mathcal{E}(N)| \ll N^{5 / 14+\varepsilon} .
$$

This completes the proof of the Theorem.

Acknowledgments. The author would like to thank the referee for his/her comments.

\section{References}

[1] S. K. K. Choi and A. V. Kumchev, Mean values of Dirichlet polynomials and applications to linear equations with prime variables, Acta Arith. 123 (2006), 125-142.

[2] G. Harman and A. V. Kumchev, On sums of squares of primes, Math. Proc. Cambridge Philos. Soc. 140 (2006), 1-13.

[3] L. K. Hua, Some results in additive prime number theory, Quart. J. Math. Oxford 9 (1938), 68-80.

[4] M. C. Leung and M. C. Liu, On generalized quadratic equations in three prime variables, Monatsh. Math. 115 (1993), 133-167.

[5] J. Y. Liu and T. Zhan, The exceptional set in Hua's theorem for three squares of primes, Acta Math. Sinica (Engl. Ser.) 21 (2005), 335-350.

[6] W. Schwarz, Zur Darstellung von Zahlen durch Summen von Primzahlpotenzen, J. Reine Angew. Math. 206 (1961), 78-112. 
[7] M. Q. Wang, On the sum of a prime and two prime squares, Acta Math. Sinica 47 (2004), 845-858 (in Chinese).

[8] M. Q. Wang and X. M. Meng, The exceptional set in the two prime squares and a prime problem, Acta Math. Sinica (Engl. Ser.) 22 (2006), 1329-1342.

[9] T. D. Wooley, Slim exceptional sets for sums of four squares, Proc. London Math. Soc. (3) 85 (2002), 1-21.

Department of Mathematics

Shanghai Jiaotong University

Shanghai 200240, People's Republic of China

E-mail: lihz@sjtu.edu.cn

Received on 9.8.2006

and in revised form on 14.4.2008 